\title{
1 Symbiosis with rhizobia limits range expansion only in polyploid legumes
}

3 Zoe A. Parshuram*1, Tia L. Harrison*1, Anna K. Simonsen², John R. Stinchcombe ${ }^{1}$, Megan E.

4 Frederickson $^{1}$

$6 *$ Authors contributed equally

$7{ }^{1}$ Department of Ecology and Evolutionary Biology, University of Toronto, Toronto, Ontario,

8 M5S 3B2 Canada

$9{ }^{2}$ Department of Biological Sciences, Florida International University, Miami, Florida, 33199,

10 United States

11

12 Author for correspondence:

13 Tia Harrison

14 Email: tia.harrison@mail.utoronto.ca

15

16 Keywords: legumes, mutualism, ploidy, range expansion, rhizobia

17 


\section{Summary}

33 - Both mutualism and polyploidy are thought to influence invasion success in plants but few studies have tested their joint effects. Mutualism can limit range expansion when plants cannot find a compatible partner in a novel habitat, or facilitate range expansion when mutualism increases a plant's niche breadth. Polyploids are also expected to have greater niche breadth because of greater self-compatibility and phenotypic plasticity, increasing invasion success.

- For 839 legume species, we compiled data from published sources to estimate ploidy, symbiotic status with rhizobia, specificity on rhizobia, and the number of introduced ranges.

- We found that diploid species have had limited spread around the globe regardless of whether they are symbiotic or how many partners of rhizobia they can host. Polyploids, in contrast, have been successfully introduced to many new ranges, but interactions with rhizobia constrain their range expansion. In a hidden state model of trait evolution, we also found evidence of a high rate of re-diploidization in symbiotic legume lineages, suggesting that symbiosis and ploidy may interact at macroevolutionary scales. 


\section{Introduction}

64 In plants, both polyploidy and mutualism are thought to contribute to ecological and evolutionary success. Polyploidization is an important driver of plant speciation, because it confers instant reproductive isolation and is associated with niche and range expansion (Soltis \& Soltis, 2016; López-Jurado et al., 2019; Sheth et al., 2020). Likewise, mutualism has been linked with high rates of lineage diversification and is thought to increase ecological opportunity by expanding niche breadth and giving rise to coevolution with mutualist partners (Gómez \& Verdú, 2012; Hembry et al., 2014; Weber \& Agrawal, 2014; Zeng \& Wiens, 2021). However, some work has also shown that engaging in mutualism can slow diversification (Kaur et al., 2019) and that relying on a mutualistic partner can limit range expansion (Simonsen et al., 2017). Despite the importance of both polyploidy and mutualism in determining where plants establish and persist, we do not know whether or how these factors interact to shape plant geographic ranges (Segraves \& Anneberg, 2016). Here, we test whether ploidy and symbiosis jointly impact invasion success in legumes.

There are several reasons why polyploids may be better invaders than diploids. Polyploids generally have greater genetic variation (Otto \& Whitton, 2000) and phenotypic plasticity (Mattingly \& Hovick, 2021), both of which may allow polyploids to establish in and rapidly adapt to novel habitats. Polyploid plants also often have higher rates of self-fertilization, a trait that is associated with greater invasion success (Barringer \& Geber, 2008). However, tests of polyploidy's effects on range size have had mixed results. In the genus Clarkia, polyploids have larger ranges than diploids (Lowry \& Lester, 2006), consistent with the above predictions. In contrast, in the Potentilleae tribe of Rosaceae, polyploids have smaller range sizes than diploids (Brittingham et al., 2018). Because species with higher ploidy tend to occupy more extreme habitats, they could become niche specialists, constraining their spread to new environments (Hummer, 2012; López-Jurado et al., 2019). Although legumes vary markedly in ploidy, no study has tested whether polyploid legumes have spread to more ranges than diploid 89 legumes.

Legumes rely on bacteria partners called rhizobia for survival when living in nutrient-

91 poor soil. Rhizobia form nodules on plant roots where rhizobia fix atmospheric nitrogen into a 92 readily available form of nitrogen for plants (van Rhijn \& Vanderleyden, 1995). The legume93 rhizobium symbiosis is over 100 million years old but nonetheless not all legumes nodulate. It is 
unclear if nodulation has evolved multiple times after an old predisposition event (Doyle, 2011; Werner et al., 2014) or if the symbiotic trait has a single evolutionary origin followed by multiple losses across the legume phylogeny (Griesmann et al., 2018).

Although mutualism can facilitate range expansion in some plant species (Afkhami et al., 2014), a previous global analysis of legumes showed that symbiosis with rhizobia limits range expansion (Simonsen et al., 2017). Legumes that depend on rhizobia for nitrogen may be unable to establish in a new range if they cannot find compatible symbionts there. Expanding on the analysis of Simonsen et al. (2017), Harrison et al. (2018) found that symbiotic legumes that associate with many rhizobia taxa have spread to more new ranges than symbiotic legumes that specialize on just one or a few rhizobia partners, again suggesting that the availability of compatible rhizobia constrains the spread of legumes around the globe. However, neither of these previous studies considered ploidy.

Ploidy is predicted to have important effects on the interaction between legumes and rhizobia. Autotetraploid plants obtain more fixed nitrogen from larger nodules compared to diploids (Forrester \& Ashman, 2020). Polyploid plants can also be more generalized on rhizobia, obtaining greater benefits from a wider diversity of rhizobia partners compared to diploids

110 (Forrester et al., 2020). Legumes vary substantially in ploidy and polyploidy influences plant 111 interactions with other species (Segraves \& Anneberg, 2016), yet we currently lack broad-scale

112 studies of how ploidy and symbiosis with rhizobia jointly influence invasion success in legumes.

113 In this study, we 1) ask whether ploidy and symbiosis interact to affect range expansion in

114 legumes and 2) estimate transition rates in symbiotic status and ploidy across the legume

115 phylogeny to better understand how the symbiosis with rhizobia evolved in plants (e.g., whether 116 it depends on evolutionary transitions in ploidy).

\section{Materials and Methods}

119 We used published data to assemble a global trait dataset that included information on ploidy, 120 mutualists, and geographic ranges for a total of 839 legume species.

122 Ploidy. We estimated ploidy (i.e., the number of copies of each chromosome in a cell) for 839 123 species of legumes using methods adapted from Brittingham et al. (2018). Specifically, we 124 extracted total chromosome count values for each species from the Chromosome Count Database 
125 (CCDB; Rice et al., 2015), the Index to Plant Chromosome Numbers (IPCN; Goldblatt \& 126 Johnson, 1979), or Rice et al. (2019). If a species was not found in one of these databases, we 127 searched Web of Science for a genus-level average for the total chromosome count number 128 (Supp Table 1). When multiple sources reported different chromosome counts for the same 129 species, we used the median value in our analyses. To calculate ploidy for each species, we 130 divided the chromosome counts by the genus-level base chromosome number as reported in 131 Bairiganjan \& Patnaik (1989) and Federov (1969). If the base chromosome number for a genus 132 was missing from the Federov (1969) table, chromosome counts were divided by the base 133 chromosome number reported for the legume subfamily: Mimosoideae ( $\mathrm{x}=13$; Santos et al., 134 2012), Caesalpiniaceae ( $\mathrm{x}=7$; Resende et al., 2013), or an average value for Papilionaceae ( $\mathrm{x}=$ 135 10.5; Lackey, 1980) to find the ploidy level of the species. We categorized all species with 136 ploidy values equal to or less than two as diploids and all species with ploidy values greater than 137 two as polyploids. Although subfamily base chromosome number and genus base chromosome 138 number values were significantly correlated in a Kendall's rank test $(\operatorname{tau}=0.413, p<0.0001)$, we 139 expect the ploidy values calculated from subfamily base chromosome numbers to be imperfect. 140 However, the dataset with ploidy calculated using only the genus-level base chromosome values 141 had fewer species overall $(\mathrm{n}=664)$ and only two species in it are non-symbiotic polyploids (Supp 142 Table 2). These two species, Cassia fistula and C. grandis, are both ornamental trees and have a 143 relatively high number of human uses in our dataset (7 uses and 4 uses, respectively) compared 144 to a mean of 2.9 human uses for symbiotic species and a mean of 1.4 uses for non-symbiotic 145 species. It is thus difficult to disentangle the influence of the number of human uses, ploidy, and 146 lack of nodules on the number of introduced ranges in these two species. Therefore, for our 147 analyses we used a combination of genus-level and subfamily-level estimated ploidy levels 148 ( $\mathrm{n}=839)$. Specifically, if ploidy calculated from the genus-level base chromosome number was 149 available, we used that value, and if it was not available, we used ploidy calculated using the 150 subfamily-level base chromosome number. Since there was a small number of polyploid species 151 calculated from subfamily base chromosome values, we searched the literature to confirm that 152 these species are indeed polyploids (Supp Table 3). Of these 62 species, 28 were confirmed to be 153 polyploids, 22 were changed to diploid in our dataset, and 12 were left as polyploid because we 154 could not find any information on ploidy level. We then chose 62 species at random from the list 155 of diploids in the dataset and looked in the literature to confirm their ploidy level. Of these 62 
species, 2 were changed to polyploid, 14 did not have ploidy levels reported in the literature, and the rest were confirmed to be diploid (Supp Table 4), suggesting that our method incorrectly classified diploids in only $\sim 3 \%$ of species. After these corrections, our dataset consisted mostly of diploid species $(n=568)$ and fewer polyploid species $(n=271$; Supp Table 2).

Mutualists. We obtained data on the symbiotic status of the legumes in our study from Werner et al. (2014). If a species is known to form nodules with rhizobia, it was categorized as a symbiotic species. We used the dataset assembled by Harrison et al. (2018) to determine whether each symbiotic legume species is a specialist or a generalist with regards to its interactions with rhizobia. Harrison et al. (2018) used data from Andrews and Andrews (2017) to determine the number of unique rhizobia genera that interact with diverse legume species. We classified a legume species as a specialist if it interacts with only one genus of rhizobia. Any legume species that interacts with more than one genus of rhizobia is a generalist.

Legume ranges. We obtained data on the number of introduced ranges for each legume species Information Service (ILDIS), Simonsen et al. (2017) classified native and invaded ranges for each species as discrete geographical ranges that roughly align with geopolitical boundaries. If a species was present in a non-native region and the neighbouring regions, it was counted as a successful invasion event, or one introduced range. We also included in our models several other covariates from the Simonsen et al. (2017) dataset, specifically the number of human uses for each legume species, the area of a species' native range, the midpoint latitude of a species' native range, and whether or not the species was annual or perennial.

Statistical analyses. All analyses were performed in R (R Core Team, 2020). We used the gls

181 function in the package nlme (Pinheiro et al., 2020) to fit phylogenetic least-squares models

182 (PGLS). We pruned the Zanne et al. (2014) angiosperm phylogeny to retain only the species in 183 our trait dataset. First, we modeled the number of introduced ranges as a function of the main 184 and interactive effects of symbiotic status (symbiotic/non-symbiotic) and ploidy

185 (diploid/polyploid). However, because the trait dataset is highly unbalanced (Supp Table 2), we 186 also split up this dataset into symbiotic species and non-symbiotic species to analyze the impact 
187 of ploidy on range expansion separately for these two categories of legumes. For only the symbiotic species in the dataset, we also modeled the main and interactive effects of specialization (specialist/generalist) and ploidy (diploid/polyploid) on introduction success. We

190 treated symbiotic status, specialization, and ploidy as binary, categorical variables. All analyses

191 included the following covariates: number of human uses, scaled value of total native area, life

192 cycle duration (annual, perennial, etc.), and absolute latitude of origin. We allowed Pagel's

193 lambda parameter to vary and optimize in the PGLS models by setting the fixed argument to

194 false. The branch lengths of the phylogeny are multiplied by this lambda parameter to account

195 for phylogenetic signal in the data. A lambda value of 0 indicates that variation in the data is

196 independent of phylogeny and a lambda value of 1 indicates a Brownian motion model of

197 evolution. We logged (base 10) the number of introduced ranges (plus one to avoid zero values)

198 in order to improve normality and homoscedasticity. When the PGLS models estimated negative

199 lambda values (suggesting phylogenetic overdispersion in the data) or values very close to zero

200 (suggesting very weak phylogenetic structure in the data) we also performed tests on the data

201 using the $g l m$ function without accounting for phylogeny. Since the number of introduced ranges

202 was not normally distributed and overdispersed, we fit the data to a quasi-poisson distribution.

203 We tested for significance by performing a type III (on models with interaction terms) or a type

204 II (on models without interaction terms) ANOVA using the Anova function in the car package

205 (Fox \& Weisberg, 2019).

207 State transitions. We modeled the evolution of ploidy and symbiosis across the Zanne et al.

208 (2014) phylogeny with the package corHMM (Beaulieu et al., 2021) to estimate transitions

209 between the following states: non-symbiotic diploid, non-symbiotic polyploid, symbiotic diploid,

210 and symbiotic polyploid. We fit a simple corHMM model with no hidden states (i.e., a single rate

211 category) and also a model with hidden states (i.e., two rate categories) on the genus/subfamily-

212 level ploidy data. Because the hidden state model always outperformed the model with no hidden

213 states, we plotted transition rates estimated from the hidden state models on the phylogeny using

214 the plotSimmap function in phytools (Revell, 2012).

215

216 Results 
217 Symbiotic status and ploidy. Non-symbiotic polyploids have been successfully introduced to

218 more new ranges than symbiotic polyploids (Fig 1a). In contrast, diploid species have been

219 introduced to few new ranges regardless of symbiotic status (Fig 1a). Symbiotic status and

220 ploidy interacted significantly to predict legume introduced ranges, although only in a non-

221 phylogenetic model (Table 1). In the PGLS model that accounts for phylogeny, there was a non-

222 significant interaction effect between symbiotic status and ploidy on the number of introduced

223 ranges (Supp Table 5). However, the PGLS model also showed a weak phylogenetic signal in the

224 number of introduced ranges $(\lambda=0.0431)$. Furthermore, when we analyzed ploidy's effects

225 separately within non-symbiotic and symbiotic legumes, we found a significant effect of ploidy

226 on range expansion only in non-symbiotic legumes (with moderate lambda value of $\lambda=0.3552$ ),

227 but not in symbiotic legumes (Supp Table 6). In our second set of analyses on symbiotic species

228 only, polyploid generalists were introduced to many more ranges than diploid generalists and

229 both diploid and polyploid specialists (Fig 1b). There was a significant interaction between

230 specialization on rhizobia and ploidy in both our phylogenetically corrected (Supp Table 5) and

231 uncorrected models (Table 1).

233 State Transitions. The hidden state model performed better $(-\operatorname{lnL}=-389.030, \mathrm{AIC}=814.060$,

$234 \mathrm{n}=839)$ than the simple model $(-\operatorname{lnL}=-439.715, \mathrm{AIC}=895.603, \mathrm{n}=839)$ therefore we present

235 results only for the hidden state model. The highest transition rate (100.00) was from polyploidy

236 to diploidy within symbiotic lineages in the first rate class (Table 2). Overall, transitions in

237 ploidy were higher within symbiotic lineages than in non-symbiotic lineages. Within non-

238 symbiotic lineages, transitions to diploidy were higher (5.0949) than transitions to polyploidy

239 (1.6291).

\section{Discussion}

242 Symbiosis and ploidy interact to affect range expansion. Overall, we found that symbiosis only

243 impacts range expansion within polyploid species and that diploid legumes spread to few new

244 ranges regardless of symbiotic status. Therefore, our results generally support the hypothesis that

245 polyploid legumes are niche generalists and better able to colonize new habitats. However, when

246 polyploids are also symbiotic they are restricted in their range expansion, suggesting that

247 symbiotic legumes have difficulty finding a compatible rhizobia partner when they are 
introduced to a novel habitat (Simonsen et al., 2017). Nonetheless, in legumes, diploidy seems to be the main factor restricting a plant's range; both non-symbiotic diploids and generalist diploids were introduced to few new ranges despite not requiring or not specializing on rhizobia. Polyploids might be good colonizers because they have more genetic material in their large genomes giving them greater adaptive potential (Otto \& Whitton, 2000). Polyploids also often have greater capacity for phenotypic plasticity and can be better competitors than diploids, since they have fast germination and growth rates (te Beest et al., 2012). However, being unable to associate with compatible rhizobia seems to cancel out the advantages of being polyploid. One explanation is that these symbiotic polyploids are largely specialists in terms of which rhizobia species they can form nodules with, making it more challenging to find beneficial rhizobia in new habitats. There were more specialist polyploids ( 25 species) than generalists ( 7 species) in our dataset, in contrast to previous results that report generalization on rhizobia in polyploids (Forrester et al., 2020). In our analyses on symbiotic species only, we found that generalist polyploids were introduced to far more ranges than any other category of legume (Fig 1b), providing further support for the theory that having many potential rhizobia partners is beneficial for range expansion, but only when paired with the benefits of being a polyploid.

Some caveats are in order, however. There were somewhat conflicting results for the interaction between symbiosis and ploidy in our PGLS analysis (non-significant) and nonphylogenetic GLM (significant). However, the PGLS model has less statistical power because it includes a phylogenetic correction, perhaps explaining the discrepancy, and the phylogenetic signal was very low in the PGLS model so phylogenetic correction may be unnecessary. We also used a combination of ploidy estimates calculated from genus- and subfamily-level base chromosome values. Although this allowed us to compile a larger dataset, it also introduced

272 which we have more accurate ploidy estimates (i.e., genus-level data) are likely well-studied 273 species that have spread widely across the globe. We corrected the data for some taxa initially 274 categorized as polyploids, and a smaller number initially categorized as diploids, by searching 275 the literature to help deal with this issue.

277 High rate of re-diploidization in legumes. Overall, the best-fitting model of trait evolution was a 278 hidden state model, likely because other unmeasured traits contribute to rate heterogeneity across 
such a large and old clade as the legumes (Beaulieu \& O'Meara, 2016). This model found that

280 evolutionary transitions in ploidy were generally higher within symbiotic than non-symbiotic

281 lineages. Within symbiotic lineages, we observed an especially high rate of re-diploidization. Re-

282 diploidization is a common process that occurs in many plant lineages (Tamayo-Ordóñez et al.,

283 2016) and seems to occur more frequently in symbiotic than non-symbiotic legume lineages.

284 Polyploid species may undergo re-diploidization if the extra genetic material in the genome

285 causes dosage imbalance, errors in mitosis or meiosis, disruption to gene regulation, or

286 epigenetic instability (Comai, 2005). Larger genomes are also generally more costly to maintain.

287 After polyploid legumes evolved genes important for interactions with rhizobia, they may have

288 experienced extensive gene loss (and thus genome reduction) during re-diploidization which

289 would reduce these costs.

290 The ancestral state in the legume tree was non-symbiotic and diploid. Our analysis

291 suggests that polyploidy directly evolved from diploidy, leading to the evolution of symbiosis

292 followed by re-diploidization in symbiotic species (Fig. 2). There is evidence in the family

293 Brassicaceae that whole genome duplication has facilitated the evolution of diverse chemical

294 defense compounds against herbivores (Edger et al., 2015). If polyploidy did indeed evolve

295 before symbiosis with rhizobia, the extra genetic material in the polyploid genome could provide

296 more opportunity for mutations and the evolution of symbiosis to occur (Soltis \& Soltis, 2016).

297 However, previous in-depth analysis of transcriptome data in a few key legume species suggests

298 that the evolution of polyploidy and symbiosis in legumes are unrelated (Cannon et al., 2010).

299 Overall, our analysis still suggests that polyploidy evolved first and is a potential predisposition

300 event to the evolution of symbiosis. However, this is an important question that warrants further

301 exploration with a larger dataset and better ploidy estimates.

303 Conclusion. Our results show the importance of considering the effects of both ploidy and

304 symbiosis on invasion success; here, we showed that ploidy can influence the impact mutualism

305 has on the spread of invasive species across the globe. Previous work has shown that symbiosis

306 (Simonsen et al., 2017) and specialization on a small number of rhizobia partners (Harrison et

$307 a l ., 2018$ ) introduces a barrier to range expansion in legumes. Our study supports these results

308 but shows that this barrier only affects polyploid legumes which otherwise have an advantage

309 over diploids in terms of successful introductions to new ranges. Looking ahead, the joint 
310 influence of ploidy and mutualism on invasion success should also be evaluated in other

311 mutualisms, such as plant-pollinator interactions; polyploids tend to be self-fertilizing, which is

312 thought to facilitate range expansion (Barringer \& Geber, 2008), but some work has suggested

313 that invasive plants can take advantage of local pollinators (Graves \& Shapiro, 2003). Our results

314 also suggest that evolutionary transitions in ploidy and symbiosis might be linked at macro-

315 evolutionary scales; polyploidy appears to have arisen before symbiosis in legumes, perhaps

316 setting the stage for the evolution of the legume-rhizobium mutualism. Nonetheless, once

317 symbiotic, legumes frequently revert to their ancestral diploid state, likely explaining why so

318 many extant symbiotic legumes are diploids.

\section{Acknowledgements}

322 We would like to thank Nikki Forrester for her methodological advice on estimating ploidy

323 levels in our global legume dataset. Our work was funded by an NSERC URSA (ZAP), an

324 NSERC PGS D scholarship and QEII-GSST award (TLH), and an NSERC Discovery Grant 325 (MEF).

\section{$\underline{\text { Data Availability }}$} publication. 
$\underline{\text { References }}$

Afkhami ME, McIntyre PJ, Strauss SY. 2014. Mutualist-mediated effects on species' range limits across large geographic scales. Ecology Letters 17: 1265-1273.

Andrews M, Andrews ME. 2017. Specificity in Legume-Rhizobia Symbioses. International Journal of Molecular Sciences 18: 705.

Bairiganjan GC, Patnaik SN. 1989. Chromosomal evolution in Fabaceae. CYTOLOGIA 54: 51-64.

Barringer BC, Geber MA. 2008. Mating System and Ploidy Influence Levels of Inbreeding Depression in Clarkia (onagraceae). Evolution 62: 1040-1051.

Beaulieu J, O'Meara B, Oliver J, Boyko J. 2021. corHMM: Hidden Markov Models of

354 Beaulieu JM, O’Meara BC. 2016. Detecting Hidden Diversification Shifts in Models of TraitDependent Speciation and Extinction. Systematic Biology 65: 583-601. interaction outcomes in a legume-rhizobium mutualism. Proceedings of the Royal Society B: Biological Sciences 288: 20202753.

Brittingham HA, Koski MH, Ashman T-L. 2018. Higher ploidy is associated with reduced range breadth in the Potentilleae tribe. American Journal of Botany 105: 700-710.

Comai L. 2005. The advantages and disadvantages of being polyploid. Nature Reviews Genetics 6: $836-846$.

365 Doyle JJ. 2011. Phylogenetic Perspectives on the Origins of Nodulation. Molecular Plant366 Microbe Interactions 24: 1289-1295.

367 Edger PP, Heidel-Fischer HM, Bekaert M, Rota J, Glöckner G, Platts AE, Heckel DG, Der

368 JP, Wafula EK, Tang M, et al. 2015. The butterfly plant arms-race escalated by gene and 369 genome duplications. Proceedings of the National Academy of Sciences 112: 8362-8366.

370 Fedorov AA. 1969. Chromosomal numbers of flowering plants. Academy of Sciences of the 371 USSR, the Komarov V.L. Botanical Institute, Nauka, Leningrad. 
372 Forrester NJ, Ashman T-L. 2020. Autopolyploidy alters nodule-level interactions in the

373 legume-rhizobium mutualism. American Journal of Botany 107: 179-185.

374 Forrester NJ, Rebolleda-Gómez M, Sachs JL, Ashman T. 2020. Polyploid plants obtain

375 greater fitness benefits from a nutrient acquisition mutualism. New Phytologist 227: 944-954.

376 Fox J, Weisberg S. 2019. An R companion to applied regression, 3rd ed. Thousand Oaks, CA:

377 Sage. URL: https://socialsciences.mcmaster.ca/jfox/Books/Companion/

378 Goldblatt P, Johnson DE. 1979. Index to plant chromosome numbers. Missouri Botanical

379 Garden, St. Louis.

380 Gómez JM, Verdú M. 2012. Mutualism with Plants Drives Primate Diversification. Systematic

381 Biology 61: 567-577.

382 Graves SD, Shapiro AM. 2003. Exotics as host plants of the California butterfly fauna.

383 Biological Conservation 110: 413-433.

384 Griesmann M, Chang Y, Liu X, Song Y, Haberer G, Crook MB, Billault-Penneteau B,

385 Lauressergues D, Keller J, Imanishi L, et al. 2018. Phylogenomics reveals multiple losses of 386 nitrogen-fixing root nodule symbiosis. Science 361: eaat1743.

387 Harrison TL, Simonsen AK, Stinchcombe JR, Frederickson ME. 2018. More partners, more 388 ranges: generalist legumes spread more easily around the globe. Biology Letters 14: 20180616.

389 Hembry DH, Yoder JB, Goodman KR. 2014. Coevolution and the Diversification of Life. The 390 American Naturalist 184: 425-438.

391 Hummer KE. 2012. A NEW SPECIES OF FRAGARIA (ROSACEAE) FROM OREGON.

392 Journal of the Botanical Research Institute of Texas 6: 9-15.

393 Kaur KM, Malé P-JG, Spence E, Gomez C, Frederickson ME. 2019. Using text-mined trait

394 data to test for cooperate-and-radiate co-evolution between ants and plants. PLOS Computational 395 Biology 15: e1007323.

396 Lackey JA. 1980. Chromosome Numbers in the Phaseoleae (Fabaceae: Faboideae) and their 397 Relation to Taxonomy. American Journal of Botany 67: 595-602.

398 López-Jurado J, Mateos-Naranjo E, Balao F. 2019. Niche divergence and limits to expansion 399 in the high polyploid Dianthus broteri complex. New Phytologist 222: 1076-1087.

400 Lowry E, Lester SE. 2006. The biogeography of plant reproduction: potential determinants of 401 species' range sizes. Journal of Biogeography 33: 1975-1982.

402 Mattingly KZ, Hovick SM. 2021. Autopolyploids of Arabidopsis thaliana are more 
phenotypically plastic than their diploid progenitors. Annals of Botany: mcab081.

Otto SP, Whitton J. 2000. Polyploid Incidence and Evolution. Annual Review of Genetics 34: $401-437$.

406 Pinheiro J, Bates D, DebRoy S, Sarkar D, R Core Team. 2020. nlme: Linear and Nonlinear

407 Mixed Effects Models. R package version 3.1-150, URL: https:/CRAN.R-

408 project.org $/$ package $=$ nlme $>$.

409 Resende KFM de, Davide LC, Torres GA. 2013. Chromosome number and meiosis in 410 populations of Senna species (Caesalpinioideae - Fabaceae) from Southeast Brazil. Caryologia 411 66: $1-5$.

412 Revell LJ. 2012. phytools: an R package for phylogenetic comparative biology (and other 413 things). Methods in Ecology and Evolution 3: 217-223.

414 van Rhijn P, Vanderleyden J. 1995. The Rhizobium-plant symbiosis. Microbiological Reviews 415 59: 124-142.

416 Rice A, Glick L, Abadi S, Einhorn M, Kopelman NM, Salman-Minkov A, Mayzel J, Chay

417 O, Mayrose I. 2015. The Chromosome Counts Database (CCDB) - a community resource of 418 plant chromosome numbers. New Phytologist 206: 19-26.

419 R Core Team. 2020. R: A language and environment for statistical computing. Vienna, Austria:

420 R Foundation for Statistical Computing. URL: https://www.R-project.org/.

421 Rice A, Šmarda P, Novosolov M, Drori M, Glick L, Sabath N, Meiri S, Belmaker J, 422 Mayrose I. 2019. The global biogeography of polyploid plants. Nature Ecology \& Evolution 3: $423265-273$.

424 Santos ECXR, Carvalho R, Almeida EM, Felix LP. 2012. Chromosome number variation and 425 evolution in Neotropical Leguminoseae (Mimosoideae) from northeastern Brazil. Genetics and 426 Molecular Research 11: 2451-2475.

427 Segraves KA, Anneberg TJ. 2016. Species interactions and plant polyploidy. American Journal 428 of Botany 103: 1326-1335.

429 Sheth SN, Morueta-Holme N, Angert AL. 2020. Determinants of geographic range size in 430 plants. New Phytologist 226: 650-665.

431 Simonsen AK, Dinnage R, Barrett LG, Prober SM, Thrall PH. 2017. Symbiosis limits 432 establishment of legumes outside their native range at a global scale. Nature Communications 8: 43314790. 
434 Soltis PS, Soltis DE. 2016. Ancient WGD events as drivers of key innovations in angiosperms.

435 Current Opinion in Plant Biology 30: 159-165.

436 Tamayo-Ordóñez MC, Espinosa-Barrera LA, Tamayo-Ordóñez YJ, Ayil-Gutiérrez B,

437 Sánchez-Teyer LF. 2016. Advances and perspectives in the generation of polyploid plant

438 species. Euphytica 209: 1-22.

439 te Beest M, Le Roux JJ, Richardson DM, Brysting AK, Suda J, Kubešová M, Pyšek P.

440 2012. The more the better? The role of polyploidy in facilitating plant invasions. Annals of

441 Botany 109: 19-45.

442 Weber MG, Agrawal AA. 2014. Defense mutualisms enhance plant diversification.

443 Proceedings of the National Academy of Sciences 111: 16442-16447.

444 Werner GDA, Cornwell WK, Sprent JI, Kattge J, Kiers ET. 2014. A single evolutionary

445 innovation drives the deep evolution of symbiotic N2-fixation in angiosperms. Nature

446 Communications 5: 4087.

447 Zanne AE, Tank DC, Cornwell WK, Eastman JM, Smith SA, FitzJohn RG, McGlinn DJ,

448 O'Meara BC, Moles AT, Reich PB, et al. 2014. Three keys to the radiation of angiosperms into 449 freezing environments. Nature 506: 89-92.

450 Zeng Y, Wiens JJ. 2021. Species interactions have predictable impacts on diversification.

451 Ecology Letters 24: 239-248. 


\section{$\bigcirc$ Diploid $\bigcirc$ Polyploid}
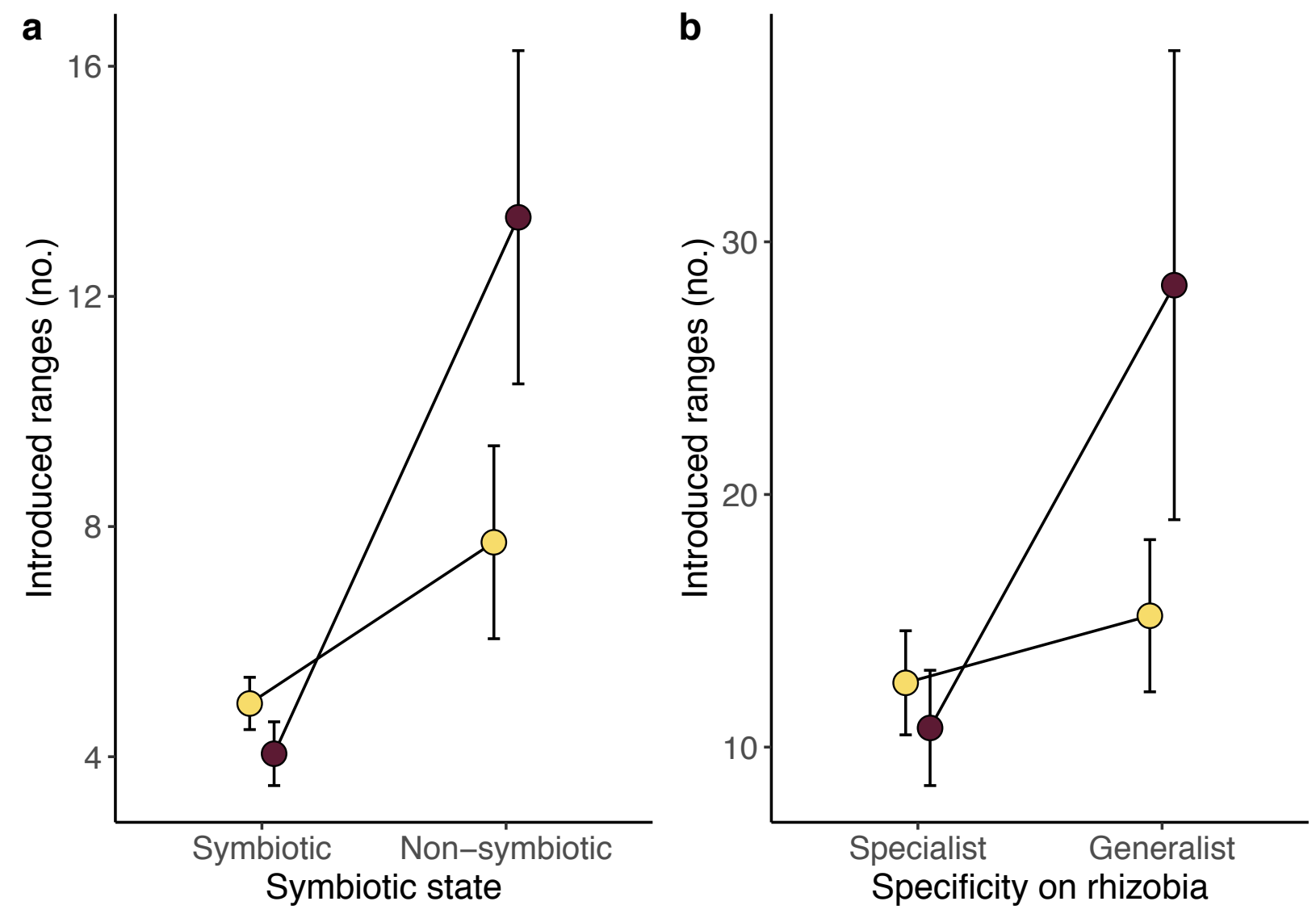

Figure 1. Mean ( $\pm 1 \mathrm{SE})$ number of introduced ranges for diploid and polyploid legumes that a) form nodules with rhizobia ('symbiotic') or do not form nodules ('non-symbiotic') and b) associate with only one genus of rhizobia ('specialist') or associate with more than one genus of rhizobia ('generalist'). 
non-symbiotic, diploid, R1 non-symbiotic, polyploid, R1 symbiotic, diploid, R1 symbiotic, polyploid, R1 non-symbiotic, diploid, R2 non-symbiotic, polyploid, R2 symbiotic, diploid, R2 symbiotic, polyploid, R2

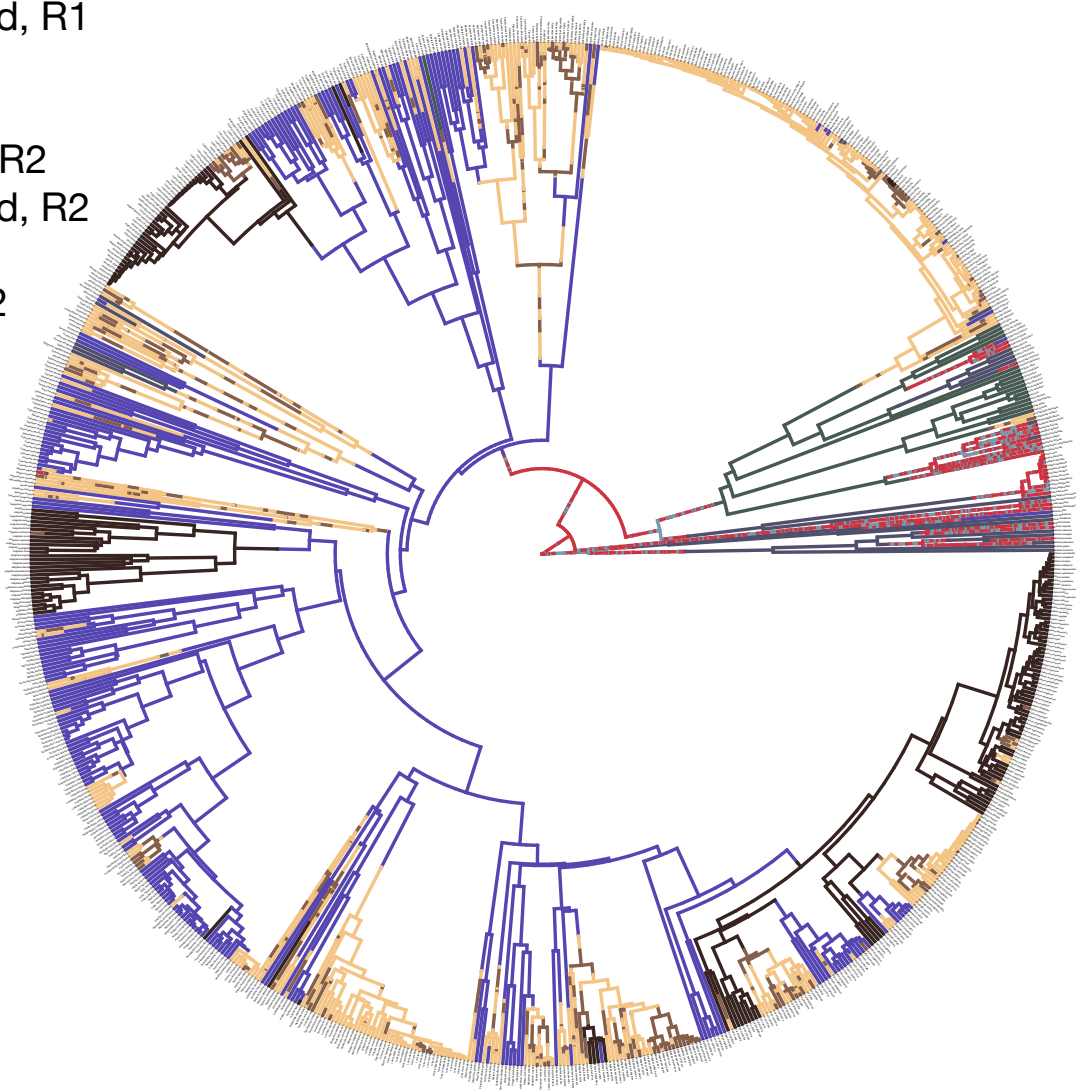

Figure 2. Transition rates between four states ('non-symbiotic diploid', 'non-symbiotic estimated from a corHMM (hidden state) model. Ploidy is estimated from combined genus and subfamily-level base chromosome numbers. 
Table 1. Estimates and results for effects of ploidy and symbiotic status on the number of introduced ranges in legumes obtained from glm models fit with a quasi-poisson distribution. Ploidy is estimated from combined genus and subfamily-level base chromosome numbers. Italicized factors are significant at $p<0.05$ and $p$ values reported here are the results of type III ANOVAs. Bolded factors highlight significant interaction effects on legume introductions. Ploidy was coded as $0=$ diploid and $1=$ polyploid while symbiotic status was coded as $0=$ nonsymbiotic and $1=$ symbiotic in the model. Therefore, the intercept represents non-symbiotic diploids. Specialists were coded as 1 and generalists as 0 in the model.

\begin{tabular}{llllll}
\hline Factor & Estimate & s.e. & d.f. & Wald $\chi^{2}$ & p value \\
\hline Symbiosis status, d.f. $=\mathbf{8 1 0}$ & & & & \\
\hline Ploidy (polyploid) & 0.573 & 0.210 & 1 & 6.530 & 0.0106 \\
Symbiosis (symbiotic) & 0.248 & 0.178 & 1 & 2.300 & 0.1295 \\
Total native area & -0.121 & 0.040 & 1 & 13.730 & 0.0002 \\
Human uses & 0.432 & 0.018 & 1 & 595.820 & $<0.0001$ \\
Absolute latitude & -0.011 & 0.004 & 1 & 12.160 & 0.0005 \\
Annual & 0.405 & 0.137 & 1 & 11.940 & 0.0005 \\
Ploidy $*$ Symbiosis & $\mathbf{- 0 . 6 4 9}$ & $\mathbf{0 . 2 4 3}$ & $\mathbf{1}$ & $\mathbf{6 . 8 6 0}$ & $\mathbf{0 . 0 0 8 8}$ \\
\hline Specialist status, d.f. $=$ & $\mathbf{1 2 9}$ & & 1 & & $<$ \\
\hline Ploidy (polyploid) & 0.498 & 0.252 & 1 & 3.114 & 0.0776 \\
Specificity (specialist) & 0.116 & 0.178 & 1 & 0.420 & 0.5167 \\
Total native area & -0.220 & 0.044 & 1 & 22.078 & $<0.0001$ \\
Human uses & 0.290 & 0.030 & 1 & 89.031 & $<0.0001$ \\
Absolute latitude & -0.020 & 0.005 & 1 & 15.868 & $<0.0001$ \\
Annual & 0.239 & 0.166 & 1 & 1.764 & 0.1841 \\
Ploidy $*$ Specificity & $\mathbf{- 0 . 9 3 1}$ & $\mathbf{0 . 3 1 2}$ & $\mathbf{1}$ & $\mathbf{6 . 6 4 0}$ & $\mathbf{0 . 0 0 9 9}$ \\
\hline & & & & & \\
\hline
\end{tabular}


518 Table 2. corHMM results for hidden state model for the genus and subfamily level ploidy 519 values. The white quadrants indicate observed state transitions within rate categories and grey 520 quadrants indicate state transitions between rate categories. The different states are represented 521 by $1=$ non-symbiotic diploid, $2=$ non-symbiotic polyploid, $3=$ symbiotic diploid, 4=symbiotic 522 polyploid. The two rate classes are represented as R1 and R2. Highest transition rates are in bold. 523 Transitions are read from row to column.

\begin{tabular}{|c|c|c|c|c|c|c|c|c|}
\hline & 1,R1 & 2,R1 & $3, \mathrm{R} 1$ & 4,R1 & $1, \mathrm{R} 2$ & 2,R2 & 3,R2 & 4,R2 \\
\hline 1,R1 & - & 1.6291 & 0.0086 & - & 0.0056 & - & - & - \\
\hline 2,R1 & 5.0949 & - & - & $<0.0001$ & - & 0.0056 & - & - \\
\hline $3, \mathrm{R} 1$ & 0.0024 & - & - & 8.5966 & - & - & 0.0056 & - \\
\hline 4,R1 & - & $<0.0001$ & 100.00 & - & - & - & - & 0.0056 \\
\hline $1, \mathrm{R} 2$ & 0.0121 & - & - & - & - & $<0.0001$ & $<0.0001$ & - \\
\hline 2,R2 & - & 0.0121 & - & - & $<0.0001$ & - & - & 0.0060 \\
\hline $3, \mathrm{R} 2$ & - & - & 0.0121 & - & $<0.0001$ & - & - & 0.2656 \\
\hline 4,R2 & - & - & - & 0.0121 & - & $<0.0001$ & 0.0351 & - \\
\hline
\end{tabular}

